\title{
The diffusion in the quantum Smoluchowski equation
}

\author{
Jerzy Łuczka ${ }^{\mathrm{a}, *}$, Ryszard Rudnicki ${ }^{\mathrm{b}, \mathrm{c}}$, Peter Hänggi ${ }^{\mathrm{d}}$ \\ anstitute of Physics, University of Silesia, 40-007 Katowice, Poland \\ ${ }^{\mathrm{b}}$ Institute of Mathematics, Polish Academy of Sciences, 40-007 Katowice, Poland \\ ${ }^{\mathrm{c}}$ Institute of Mathematics, University of Silesia, 40-007 Katowice, Poland \\ ${ }^{\mathrm{d}}$ Institute of Physics, University of Augsburg, Universitätsstrasse 1, D-86135 Augsburg, Germany
}

Received 23 September 2004

Available online 1 January 2005

\begin{abstract}
A novel quantum Smoluchowski dynamics in an external, nonlinear potential has been derived recently. In its original form, this overdamped quantum dynamics is not compatible with the second law of thermodynamics if applied to periodic, but asymmetric ratchet potentials. An improved version of the quantum Smoluchowski equation with a modified diffusion function has been put forward in L. Machura et al. (Phys. Rev. E 70 (2004) 031107) and applied to study quantum Brownian motors in overdamped, arbitrarily shaped ratchet potentials. With this work we prove that the proposed diffusion function, which is assumed to depend (in the limit of strong friction) on the second-order derivative of the potential, is uniquely determined from the validity of the second law of thermodynamics in thermal, undriven equilibrium. Put differently, no approximation-induced quantum Maxwell demon is operating in thermal equilibrium. Furthermore, the leading quantum corrections correctly render the dissipative quantum equilibrium state, which distinctly differs from the corresponding Gibbs state that characterizes the weak (vanishing) coupling limit.
\end{abstract}

(C) 2005 Elsevier B.V. All rights reserved.

PACS: 05.40.-a; 02.50.Ey; 05.10.Gg; 05.60.Gg

Keywords: Smoluchowski equation; Large friction; Quantum corrections; Brownian motors; Dissipative quantum dynamics

\footnotetext{
*Corresponding author. Tel.: + 48323591173.

E-mail address: luczka@us.edu.pl (J. Łuczka).
} 


\section{Introduction}

In classical statistical physics, the description of a system coupled to thermal bath of temperature $T$ is formulated in terms of Langevin-type equations and corresponding Fokker-Planck or master equations [1,2]. This scheme models the phenomenon of Brownian motion, a phenomenon that has been described theoretically by the two "grandfathers" of Brownian motion: Albert Einstein and Marian von Smoluchowski [3,4]. For a classical Brownian particle moving in an external field, the statistical properties are described by the Klein-Kramers equation in the phase space of position and momentum degree of freedom $[1,2,5]$. In the strong friction limit it reduces to the so-called Smoluchowski equation in position space alone [6]. The issue of quantum Brownian motion is more subtle [7]. In this context, it is important to note that for a correct description of the quantum dynamics of the subsystem, the Brownian particle must at all times be consistent with the Heisenberg principle and the commutator structure of quantum dynamics; as a consequence, the reduced dynamics modeled in the form of a corresponding quantum Langevin equation must necessarily operate in the total Hilbert space of the system dynamics (i.e., the particle) and the thermal bath degrees of freedom. Given an initial preparation scheme [8], a consistent statistical description is also possible as well in terms of (generalized) quantum master equations for the reduced density operator of the system dynamics alone, i.e., the quantum Brownian dynamics. An example constitutes the weak coupling limit, for which quantum master equations (e.g. of Lindblad form) have been derived [9]. The strong friction limit has only recently attracted interest. Different approaches have been proposed in the recent years that are seemingly not wholly consistent with each other [10]. Here, we follow the scheme that is rigorously based on a path integral formulation of the (reduced) quantum Brownian motion [5,7].

Following Ankerhold-Pechukas-Grabert [11], the limit of strong friction can be described by a corresponding quantum Smoluchowski dynamics containing leading quantum corrections. For a particle of mass $M$ moving in the potential $V(x)$, such a quantum Smoluchowski equation has been derived for the diagonal part of the density operator $\rho(t)$, i.e., for the probability density $P(x, t)=\langle x|\rho(t)| x\rangle$ in the position space $x$. It explicitly reads [11]

$$
\gamma M \frac{\partial}{\partial t} P(x, t)=\frac{\partial}{\partial x} V_{e f f}^{\prime}(x) P(x, t)+\frac{\partial^{2}}{\partial x^{2}} D_{e f f}(x) P(x, t),
$$

where $\gamma$ is a friction coefficient. The effective potential reads

$$
V_{e f f}(x)=V(x)+(1 / 2) \lambda V^{\prime \prime}(x),
$$

wherein the prime denotes the derivative with respect to the coordinate $x$. The effective diffusion coefficient

$$
D_{e f f}(x)=D_{1}(x)=\frac{1}{\beta}\left[1+\lambda \beta V^{\prime \prime}(x)\right]
$$


both includes leading quantum corrections described by the (quantum)-parameter

$$
\lambda=(\hbar / \pi M \gamma) \ln (\hbar \beta \gamma / 2 \pi), \quad \beta=1 / k_{B} T
$$

and $k_{B}$ is the Boltzmann constant. The parameter $\lambda$ is nonlinear with respect to the Planck constant $\hbar$ and measures typical quantum fluctuations in the position space $[11,12]$. Note that at strong friction, the leading quantum corrections vanish for (biased) free quantum-Brownian motion, i.e., for potentials with $V(x)=$ const, or with linear $V(x)=\alpha x$; in the two latter cases no normalizable stationary state occurs. The validity regime for Eq. (1) is in the quantum Smoluchowski regime when $\lambda \gg 1 / M \beta \gamma^{2}$ and $k_{B} T \ll \hbar \gamma$ [11]. Note that formally $\lambda$ diverges at zero temperature; it is thus important to note that the limit $\beta \rightarrow \infty$ should be taken only after the limit $\gamma \rightarrow \infty$.

This equation assumes the form - within the regime of validity with $D_{\text {eff }}>0$ - of a classical Smoluchowski equation with state-dependent diffusion. Thus, it intrinsically obeys detailed balance for all confining potentials $V_{\text {eff }}(x)$ obeying $V_{\text {eff }}(x \rightarrow$ $\pm)=\infty$. Put differently, for unbounded, confining potentials this one-dimensional Smoluchowski equation obeys formally detailed balance. This situation changes, however, for the class of bounded potentials. For example, in a periodic potential $V_{\text {eff }}(x)$, the second law of thermodynamics implies that no stationary current is supported. This is the case if detailed balance also holds for the class of such periodic potentials, or more generally, for bounded potentials.

It is thus a nontrivial observation that-upon inspection-the above Smoluchowski equation indeed does not obey the second law of thermodynamics for periodic potentials. Indeed, let us consider a periodic potential $V(x)=V(x+L)$ of period $L$. From Eq. (1), following the reasoning of Stratonovich [13], but generalized here to state-dependent diffusion, see [14], the stationary drift velocity $\langle v\rangle$ of the particle emerges as

$$
\langle v\rangle=\frac{L}{\gamma M} \frac{1-\exp [\Psi(L)]}{\int_{0}^{L} D_{1}^{-1}(x) \exp [-\Psi(x)] \int_{x}^{x+L} \exp [\Psi(y)] \mathrm{d} y \mathrm{~d} x}
$$

with the generalized thermodynamic potential given by

$$
\Psi(x)=\int_{0}^{x} \frac{V_{e f f}^{\prime}(y)}{D_{e f f}(y)} \mathrm{d} y .
$$

If the periodic potential is asymmetric, e.g., $V(x)=\cos (2 \pi x)+(1 / 4) \sin (4 \pi x)$, and $L=1$, the generalized thermodynamic potential $\Psi(L) \neq 0$. Consequently, a nonzero stationary drift velocity occurs in the thermal equilibrium state, i.e., an approximation-induced quantum Maxwell demon [15] is seemingly at work.

\section{Diffusion function}

In the recent work [16], we have proposed an improved quantum Smoluchowski equation, in which the effective potential $V_{\text {eff }}(x)$ is the same as in (2) but the diffusion function $D_{e f f}(x)$ is modified in such a way that the modified Smoluchowski 
equation no longer causes such a perpetual motion phenomenon. This new quantum Smoluchowski dynamics is subject to the following construction criteria:

(i) Remedy the diffusion function to obey $\Psi(L) \equiv 0$ for any periodic potential $V(x)$.

(ii) Assume that the diffusion function is a function $F$ of the second-order derivative of $V(x)$, i.e., $D_{\text {eff }}(x)=F\left[V^{\prime \prime}(x)\right]$, as in (3).

(iii) For the harmonic oscillator, the position equilibrium probability emerging from (1) should coincide at strong friction with the exact distribution [17].

Condition (ii) may seem to be restrictive: the unknown diffusion function could depend functionally not only on $V^{\prime \prime}(x)$, but also on higher order derivatives of the potential. However, if we take into account the leading quantum corrections in the asymptotic strong friction limit, such higher order derivatives of $V(x)$ can safely be neglected.

Next, from the first two conditions we obtain

$$
\int_{0}^{L} \frac{V^{\prime}(x)+(1 / 2) \lambda V^{\prime \prime \prime}(x)}{F\left[V^{\prime \prime}(x)\right]} \mathrm{d} x=0 .
$$

The second part of the integral, which contains the contribution $V^{\prime \prime \prime}(x)$, is zero for arbitrary periodic functions $V(x)$ and functions $F\left[V^{\prime \prime}(x)\right]$, for which the integral exists. Indeed, upon a change in the integration variable $x$ to the new variable $y=V^{\prime \prime}(x)$, and a partition of the interval $[0, L]$ into a sum of subintervals, on which the function $V^{\prime \prime}(x)$ is monotonic, we find that this second contribution is proportional to

$$
\begin{aligned}
\sum_{i=0}^{n-1} \int_{y_{i}}^{y_{i+1}}(1 / F(y)) \mathrm{d} y & =\left.\sum_{i=0}^{n-1} A(y)\right|_{y_{i}} ^{y_{i+1}} \\
& =A\left(y_{n}\right)-A\left(y_{0}\right)=A\left(V^{\prime \prime}(L)\right)-A\left(V^{\prime \prime}(0)\right)=0,
\end{aligned}
$$

because $V^{\prime \prime}(x)$ is periodic and $V^{\prime \prime}(0)=V^{\prime \prime}(L)$. The first position is $y_{0}=V^{\prime \prime}(0)$, the last one is $y_{n}=V^{\prime \prime}(L)$, and the remaining positions are $y_{i}=V^{\prime \prime}\left(x_{i}\right)$, where the set of positions $x_{i}$ are positions of extremal values of the function $V^{\prime \prime}(x)$. The function $A(y)$ denotes the anti-derivative (i.e., the integral) of $1 / F(y)$. Therefore, condition (7) becomes equivalent to

$$
\int_{0}^{L} \frac{V^{\prime}(x)}{F\left(V^{\prime \prime}(x)\right)} \mathrm{d} x=0 .
$$

With this work we show that there is indeed only one function $F(y)$, for which condition (9) is obeyed for an arbitrary periodic potential $V(x)=V(x+L)$. In our proof, we use the well-known functional method familiar from theoretical mechanics: among all possible trajectories, find the one for which the functional (called the action) is extremal. Our task can be formulated as follows: among all possible functions $F\left(V^{\prime \prime}(x)\right)$, find the one for which (9) is fulfilled. To this goal, let us denote

$$
f(x)=V^{\prime}(x), \quad G(y)=1 / F(y) .
$$


Condition (9) then takes on the form

$$
\int_{0}^{L} G\left(f^{\prime}(x)\right) f(x) \mathrm{d} x=0 .
$$

Let $X$ be a set of $L$-periodic functions $f: \mathbb{R} \rightarrow \mathbb{R}$ such that $\int_{0}^{L} f(x) \mathrm{d} x=0$. Moreover, mainly for technical reasons, we assume from here on that $f \in C^{3}$. Next, we derive the following theorem:

Theorem 1. Let $G: \mathbb{R} \rightarrow \mathbb{R}$ be a three times differentiable function with continuous derivative $G^{\prime \prime \prime}$. If (11) holds for every $f \in X$, then $G$ is a linear function, i.e., $G(y)=$ $a+b y$.

Proof. Consider a functional $S$ on $X$ given by the expression

$$
S(f)=\int_{0}^{L} G\left(f^{\prime}(x)\right) f(x) \mathrm{d} x .
$$

Then, from (11) it follows that the functional $S(f)$ is constant and its increment $S(f+h)-S(f)=0$ for every $f \in X$ and $h \in X$; this implies that the functional derivative $(\delta S / \delta f)(h)=0$, i.e., by use of the notation $G\left(f^{\prime}(x)\right)=\left(G \circ f^{\prime}\right)(x)$ we obtain

$$
\begin{aligned}
\frac{\delta S}{\delta f}(h)= & \int_{0}^{L}\left[\left(G^{\prime} \circ f^{\prime}\right)(x) f(x) h^{\prime}(x)+\left(G \circ f^{\prime}\right)(x) h(x)\right] \mathrm{d} x \\
= & \left(G^{\prime} \circ f^{\prime}\right)(L) f(L) h(L)-\left(G^{\prime} \circ f^{\prime}\right)(0) f(0) h(0) \\
& +\int_{0}^{L}\left\{\left(G \circ f^{\prime}\right)(x)-\left[\left(G^{\prime} \circ f^{\prime}\right)(x) f(x)\right]^{\prime}\right\} h(x) \mathrm{d} x .
\end{aligned}
$$

Since $f(0)=f(L), h(0)=h(L)$ and $f^{\prime}(L)=f^{\prime}(0)$, one finds

$$
\frac{\delta S}{\delta f}(h)=-\int_{0}^{L} \psi(x) h(x) \mathrm{d} x=0,
$$

where

$$
\psi(x)=\left[\left(G^{\prime} \circ f^{\prime}\right)(x) f(x)\right]^{\prime}-\left(G \circ f^{\prime}\right)(x) .
$$

Given the proposition proved in the appendix it follows that $\psi \equiv$ const. Next, let $f_{\alpha, \mu}$ be a family of functions from the set $X$ such that $f_{\alpha, \mu}(y)=\frac{\mu}{2} y^{2}+\alpha, \alpha \in \mathbb{R}, \mu \in \mathbb{R}$, for $y \in\left(\frac{L}{3}, \frac{2 L}{3}\right)$. After substituting $f_{\alpha, \mu}$ into (17) we obtain

$$
\left[G^{\prime}(\mu y)\left(\frac{\mu}{2} y^{2}+\alpha\right)\right]^{\prime}-G(\mu y) \equiv \text { const },
$$

and consequently

$$
\alpha \mu G^{\prime \prime}(\mu y)+\frac{\mu^{2}}{2} y^{2} G^{\prime \prime}(\mu y)+\mu y G^{\prime}(\mu y)-G(\mu y) \equiv \text { const } .
$$

Since the left-hand side of this equation is constant for arbitrary $\alpha$, we conclude that $G^{\prime \prime}(\mu y) \equiv$ const for $\mu y \in\left(\frac{\mu L}{3}, \frac{2 \mu L}{3}\right)$. Since $\mu$ is an arbitrary constant we have $G^{\prime \prime} \equiv$ const 
on the whole $\mathbb{R}$. Consequently,

$$
\frac{1}{2}(\mu y)^{2} G^{\prime \prime}(\mu y)+\mu y G^{\prime}(\mu y)-G(\mu y) \equiv \text { const }
$$

for $y \in\left(\frac{L}{3}, \frac{2 L}{3}\right)$. Since $\mu$ is an arbitrary constant we also obtain that

$$
\frac{1}{2} y^{2} G^{\prime \prime}(y)+y G^{\prime}(y)-G(y) \equiv \text { const }
$$

for $y \in \mathbb{R}$. With the result $G^{\prime \prime}=$ const, it thus follows uniquely that $G(y)=a+b y+$ $c y^{2}$, where $a, b, c \in \mathbb{R}$. Insertion of $G(y)$ into (21) yields $c=0$. As a main result we obtain $G(y)=a+b y$, thereby completing the proof.

\section{Discussion and conclusion}

Given Theorem 1, it follows that the sought-after diffusion function $F$ in (9) assumes the form

$$
F\left[V^{\prime \prime}(x)\right]=\frac{1}{a+b V^{\prime \prime}(x)},
$$

with arbitrary constants $a$ and $b$. These two constants can be determined as follows: expand (22) into a series. Then for $a=\beta$ and $b=-\lambda \beta^{2}$, the diffusion functions (3) and (22) coincide to the first order with respect to the quantity $\varepsilon(x)=\left|\lambda \beta V^{\prime \prime}(x)\right|<1$. For smooth periodic functions $V(x)$, this inequality can be fulfilled for arbitrary $x$ and sufficiently small $\lambda \beta$. Note that, $V^{\prime \prime}(x)$ is bounded for arbitrary $x$ and the convergence of (22) to Eq. (3) is uniform. Given the above findings, we propose a new quantum Smoluchowski equation (1) with the modified diffusion function reading

$$
D_{e f f}(x)=D_{2}(x)=\frac{1}{\beta\left[1-\lambda \beta V^{\prime \prime}(x)\right]},
$$

which must be interpreted as the leading, nonperturbative, asymptotic large friction result.

In conclusion, the above quantum stochastic dynamics becomes, in this strong friction limit equivalent to a classical, overdamped Brownian dynamics in the effective potential (2) and with a state-dependent diffusion coefficient (23). The corresponding classical Langevin equation reads in the Ito-representation [1]

$$
\gamma M \dot{x}=-V_{e f f}^{\prime}(x)+\sqrt{2 \gamma M D_{2}(x)} \xi(t),
$$

where the dot denotes the time derivative and $\xi(t)$ is classical Gaussian white noise of vanishing mean and correlation $\langle\xi(t) \xi(s)\rangle=\delta(t-s)$.

Our result for the quantum Smoluchowski equation resembles in spirit the problem of modeling colored (or correlated) noise in classical, nonlinear stochastic physics in terms of an effective Fokker-Planck equation [18]. The Langevin equation with Gaussian nonwhite noise constitutes a nonMarkovian process whose single time stochastic dynamics in limiting cases can be approximated well by a corresponding (single time) Markovian diffusion process. This latter Markovian 
diffusion process is equivalently described by the Langevin equation with Gaussian white noise and a state-dependent diffusion coefficient [18]. For example, for a Langevin equation with additive Ornstein-Uhlenbeck noise, Fox [19] derived in the limit of small Ornstein-Uhlenbeck noise correlation time $\tau$ the following asymptotic form of the diffusion function

$$
D(x)=\frac{D_{0}}{1+\tau V^{\prime \prime}(x)},
$$

where $D_{0}$ is the noise intensity. The same characteristic contribution $1 /\left[1+\tau V^{\prime \prime}(x)\right]$ occurs in the theory of unified colored-noise approximation, which in fact bridges small correlation times with large correlation times [20]. We observe that in both diffusion functions (23) and (25) the function $V^{\prime \prime}(x)$ occurs. If the correlation time $\tau \rightarrow 0$ then clearly $D(x) \rightarrow D_{0}$.

In the quantum Smoluchowski case, the corresponding thermal quantum noise is also not white and the corresponding quantum Brownian motion corresponds to a nonMarkovian quantum dynamics, which here (limit of large friction) is approximated by the Markovian diffusion process described by Eq. (1). If the analogous parameter to $\tau$, i.e., $\lambda$ approaches zero at high temperatures, i.e., $\beta \rightarrow 0$ then the new diffusion function $D_{2}(x) \rightarrow 1 / \beta$ in (23), i.e., the classical limit is approached.

The periodic stationary solution $P(x)$ of (1) with the diffusion function (23) reads

$$
P(x)=N \mathrm{e}^{-\beta \Phi(x)},
$$

where the equilibrium thermodynamic potential assumes the form

$$
\Phi(x)=V(x)+\frac{1}{2} \lambda V^{\prime \prime}(x)-\frac{1}{2} \lambda \beta\left[V^{\prime}(x)\right]^{2}-\frac{1}{4} \lambda^{2} \beta\left[V^{\prime \prime}(x)\right]^{2}-\frac{1}{\beta} \ln \left[1-\lambda \beta V^{\prime \prime}(x)\right] .
$$

To the first order in $\lambda$, it coincides with that presented in Eq. (11) of Ref. [11] and it reduces for the harmonic oscillator to the exact probability density in the strong friction limit [17]. Remarkably, this quantum equilibrium potential depends in the strong coupling limit between system and bath explicitly on the friction strength $\gamma$. Put differently, in clear contrast to the case of weak coupling, the quantum thermal equilibrium state is not of the Gibbs form, meaning that $\Phi(x) \neq V(x)$.

In summary, we have shown that the diffusion function in the quantum Smoluchowski limit is uniquely determined to be of the form in (23). It depends on the potential via $V^{\prime \prime}(x)$, and is consistent with dissipative quantum equilibrium thermodynamics. Moreover, the diffusion function should not become negative; the diffusion function in (23) is positive for the sufficiently small quantum correction parameter $\lambda$ and for bounded $V^{\prime \prime}(x)$. This holds true for the case of smooth, spatially periodic asymmetric potentials, i.e., so-called Brownian motor systems [21], for which our approximate quantum Smoluchowski equation is valid in the whole state space of the system [16]. If $V^{\prime \prime}(x)$ is not bounded, as it occurs for bistable systems, the Smoluchowski equation (1) with the new diffusion function (23) is valid only in a restricted domain of the state space. This same restriction holds also for the related problem of colored noise-driven classical dynamical systems [18]. 


\section{Acknowledgements}

Support by the European Science Foundation (Stochastic Dynamics: Fundamentals and Applications) (J.Ł.,P.H.), the Deutsche Forschungsgemeinschaft with the collaborative research centre SFB-486 (P.H.), DFG Grant Ha 1517/13-4 (P.H.), the DAAD-KBN (Stochastic Complexity) (J.Ł.,P.H.), the Foundation for Polish Science (P.H.) and the KBN Grant 2 P03A 03125 (R.R.) is gratefully acknowledged.

\section{Appendix A}

In this appendix we prove the following proposition:

Proposition 1. Let

$$
\int_{0}^{L} \psi(x) h(x) \mathrm{d} x=0
$$

for $h \in X$. Then $\psi=$ const.

Proof (indirectly). Assume that $\psi$ is not a constant function. Since we have assumed in the Theorem that the functions are in $C^{\prime \prime \prime}$, cf. (17), there exists a position $x_{0} \in$ $(0, L)$ such that $\psi^{\prime}\left(x_{0}\right) \neq 0$. Without loss of generality we can assume that $\psi^{\prime}\left(x_{0}\right)>0$, because we can replace $\psi$ by $-\psi$. Since $\psi^{\prime}$ is a continuous function, we can find $\varepsilon>0$ such that $0<x_{0}-\varepsilon<x_{0}+\varepsilon<L$ and $\psi^{\prime}(x)>0$ for $x \in\left(x_{0}-\varepsilon, x_{0}+\varepsilon\right)$. Since $\psi(x)=$ $\psi\left(x_{0}\right)+\psi^{\prime}(\theta)\left(x-x_{0}\right)$, where $\theta \in\left(x_{0}-\varepsilon, x_{0}+\varepsilon\right)$, we have $\psi(x)>\psi\left(x_{0}\right)$ for $x \in$ $\left(x_{0}, x_{0}+\varepsilon\right)$ and $\psi(x)<\psi\left(x_{0}\right)$ for $x \in\left(x_{0}-\varepsilon, x_{0}\right)$. Next, let

$$
h(x)=\left(x-x_{0}\right)^{5}\left(x-x_{0}-\varepsilon\right)^{4}\left(x-x_{0}+\varepsilon\right)^{4}
$$

for $x \in\left(x_{0}-\varepsilon, x_{0}+\varepsilon\right)$ and $h(x)=0$ for $x \in\left[0, x_{0}-\varepsilon\right] \cup\left[x_{0}+\varepsilon, L\right]$. Then $h$ is a $C^{3}-$ function and since $h(x)=0$ for $x$ in the neighborhoods of 0 and $L$, the function $h$ can be extended to a $L$-periodic function. Moreover, $h\left(x_{0}+x\right)=-h\left(x_{0}-x\right)$ which implies that $\int_{0}^{L} h(x) \mathrm{d} x=0$. This also means that $h \in X$. Next, observe that

$$
\begin{aligned}
\int_{0}^{L} \psi(x) h(x) \mathrm{d} x & =\int_{x_{0}-\varepsilon}^{x_{0}} \psi(x) h(x) \mathrm{d} x+\int_{x_{0}}^{x_{0}+\varepsilon} \psi(x) h(x) \mathrm{d} x \\
& >\int_{x_{0}-\varepsilon}^{x_{0}} \psi\left(x_{0}\right) h(x) \mathrm{d} x+\int_{x_{0}}^{x_{0}+\varepsilon} \psi\left(x_{0}\right) h(x) \mathrm{d} x \\
& =\psi_{0}(x) \int_{0}^{L} h(x) \mathrm{d} x=0
\end{aligned}
$$

because $\psi(x)>\psi\left(x_{0}\right), h(x)>0$ for $x \in\left(x_{0}, x_{0}+\varepsilon\right)$, and $\psi(x)<\psi\left(x_{0}\right), h(x)<0$ for $x \in$ $\left(x_{0}-\varepsilon, x_{0}\right)$. Consequently, $\int_{0}^{L} \psi(x) h(x) \mathrm{d} x>0$; this finding in turn contradicts the assumption that the integral is zero. Thus, for every function $f \in X$ we have $\psi=$ const, and this completes the proof of the proposition. 


\section{References}

[1] P. Hänggi, H. Thomas, Phys. Rep. 88 (1982) 207.

[2] H. Risken, The Fokker-Planck Equation, Springer, Berlin, 1996.

[3] A. Einstein, Ann. Physik (Leipzig) 17 (1905) 549;

A. Einstein, Ann. Physik (Leipzig) 19 (1906) 371;

M. Smoluchowski, Ann. Physik (Leipzig) 21 (1906) 756.

[4] A. Einstein, Naturwissenschaften 50 (1917) 107; Marian von Smoluchowski, an obituary by A. Einstein.

[5] P. Hänggi, J. Stat. Phys. 42 (1986) 105;

P. Hänggi, J. Stat. Phys. 44 (1986) 1003 (Addendum);

P. Hänggi, P. Talkner, M. Borkovec, Rev. Mod. Phys. 62 (1990) 251.

[6] M. Smoluchowski, Bull. Int. l'Acad. Sci. Cracovie, Math-Naturw. Klasse A (1913) 418-434;

Reprinted in: Ostwalds Klas. Exakten Nat. vol. 207 (2001) 25-39;

M. Smoluchowski, Ann. Physik (Leipzig) 48 (1915) 1103.

[7] H. Grabert, P. Schramm, G.-L. Ingold, Phys. Rep. 168 (1988) 1988.

[8] K.M. Fonseca-Romero, P. Talkner, P. Hänggi, Phys. Rev. A 69 (2004) 052109.

[9] G. Lindblad, Commun. Math. Phys. 48 (1976) 119;

R. Alicki, K. Lendi, Quantum Dynamical Semigroups and Applications, Springer, Berlin, 1982;

H. Spohn, Rev. Mod. Phys. 52 (1980) 569.

[10] P. Pechukas, J. Ankerhold, H. Grabert, Ann. Physik (Leipzig) 9 (2000) 794;

B. Vacchini, Phys. Rev. E 66 (2002) 027107;

D. Banerjee, B.C. Bag, S.K. Banik, D.S. Ray, Physica A 318 (2003) 6.

[11] J. Ankerhold, P. Pechukas, H. Grabert, Phys. Rev. Lett. 87 (2001) 086802.

[12] J. Ankerhold, Acta Phys. Polon. B 34 (2003) 3569.

[13] R.L. Stratonovich, Radiotekh. Electron. 3 (1958) 497;

English translation in: P.I. Kuznetsov, R.L. Stratonovich, V.I. Tikhonov (Eds.), Nonlinear Transformations of Stochastic Processes, Pergamon Press, Oxford, 1965.

[14] J. Łuczka, R. Bartussek, P. Hänggi, Europhys. Lett. 31 (1995) 431.

[15] H.S. Leff, A.F. Rex, Maxwells Demon, Entropy, Information, Computing, Adam Hilger, Bristol, 1990.

[16] L. Machura, M. Kostur, P. Hänggi, P. Talkner, J. Łuczka, Phys. Rev. E 70 (2004) 031107.

[17] H. Grabert, U. Weiss, P. Talkner, Z. Phys. B 55 (1984) 87;

P. Riseborough, P. Hänggi, U. Weiss, Phys. Rev. A 31 (1985) 471.

[18] P. Jung, P. Hänggi, Adv. Chem. Phys. 89 (1995) 239.

[19] R.F. Fox, Phys. Rev. A 34 (1986) 4525.

[20] P. Jung, P. Hänggi, Phys. Rev. A 35 (1987) 4464.

[21] P. Hänggi, R. Bartussek, Lect. Notes Phys. 476 (1996) 294;

R.D. Astumian, P. Hänggi, Phys. Today 55 (11) (2002) 33;

P. Reimann, P. Hänggi, Appl. Phys. A 75 (2002) 169;

P. Reimann, Phys. Rep. 361 (2002) 57;

H. Linke, Appl. Phys. A 75 (2002) 167, special issue on Brownian motors. 